\title{
A Retinex image enhancement based on L channel illumination estimation and gamma function
}

\author{
Yulei Huang, Yan Li, Yan Zhang \\ School of Intelligence Science and Information Engineering \\ Xi'an Peihua University, Xi'an, China \\ huangyulei@peihua.cn,79132181@qq.com, 410325568@qq.com
}

Keywords: Low illumination; Color image enhancement; L channel; Gamma function

\begin{abstract}
In order to improve the contrast of low illumination image, enhance the processing effect and eliminate artifacts, a color Retinex image enhancement algorithm based on L channel illumination estimation and two dimensional gamma functions is proposed. Firstly, the L image intensity is regarded as the product of correlation reflectivity and illumination through Lab color space transformation, and the intensity of global illumination is estimated by bilateral filtering. Then, two dimensional gamma functions are used to complete the pixel mapping, and the local details can be adaptively adjusted. Experimental results show that compared with other Retinex image enhancement algorithms, the proposed algorithm enhances the effect more naturally and produces no enhancement artifacts, which can effectively preserve the local details of the image.
\end{abstract}

\section{Introduction}

In light of insufficient lighting conditions or performance limitations of photography equipment, there will be a large number of low-illumination images in daily life and work. At this time, there is an urgent need to enhance low-light images. In order to improve the visual effect of the image and increase the visual information of the image, the image needs to be enhanced. For color images, the amount of information they contain is more abundant than gray scale images, so the corresponding enhancement processing algorithm will be more complex [1].

Contrast enhancement algorithm is one of the hot topics in image processing. Most of the traditional contrast enhancement algorithms are widely used Retinex theory[1,2]. Jobson et al. proposed a single-scale Retinex (SSR) method for image enhancement [3]. This method uses Gaussian filtering to evaluate and process the luminance of the input image. However, in the enhanced output image, halo artifacts often appear in local images with complex texture edge information, which is called color shift [4-6].

According to the principle of mimicking the operation of the human visual system, an enhanced algorithm based on Retinex can be used to enhance the output image with higher naturalness. Therefore, many researchers have recently proposed various enhancement algorithms based on Retinex [7-9]. The literature [10] uses bright pass filters and double logarithmic conversions to simultaneously process reflectivity and luminance, balancing the resulting image details and naturalness. However, none of these algorithms can meet the requirements of high naturalness and artifact-free phenomena. In order to maintain its original brightness and local details in enhanced low-light images, we need to do local adaptive brightness processing.

Based on the above research and analysis, this paper presents a color Retinex image enhancement algorithm based on L-channel illumination estimation and two-dimensional gamma function. First, the algorithm obtains L-channel components through Lab color space transformation, and uses bilateral filtering to estimate the global illumination brightness. Then, pixel mapping is performed by using a two-dimensional gamma function to achieve local brightness adaptive adjustment. Finally, the enhanced global luminance component and adaptively adjusted local luminance are combined to generate the final enhanced image. Experimental results show that compared with other Retinex image enhancement algorithms, the proposed algorithm can maintain 
the local detail texture of the enhanced image better, with higher color constancy, higher contrast and no artifact.

\section{Retinex Image Enhancement Principle Analysis}

According to the traditional Retinex principle, we set the intensity of the input image to be obtained by combining the background reflectance with the luminance. The formula is as follows:

$$
I(x, y)=R(x, y) \cdot L(x, y)
$$

According to the principle of the single-scale Retinex algorithm, the input image is processed with a Gaussian filter to obtain the estimated value, and the scene reflectivity is obtained. The calculation method is as follows:

$$
\begin{gathered}
R_{S S R}(x, y)=\log \mathrm{I}(x, y)-\log \{F(x, y) * I(x, y)\} \\
F(x, y)=K \cdot \exp \left(-\frac{x^{2}+y^{2}}{k^{2}}\right)
\end{gathered}
$$

Where, $F(x, y)$ is representative of a typical normalized Gaussian function[2], which $K$ is a normalized parameter factor that meets the condition $\sum_{x} \sum_{y} F(x, y)=1$.

In order to avoid artifacts, a multi-scale MSR algorithm was proposed in [3]. The resulting scene reflectance is a weighted sum of multiple image dimensions. Its expression formula is:

$$
R_{M S R}(x, y)=\sum_{n=1}^{N} w_{n} \cdot R_{S S R, n}(x, y)
$$

Among them, $N$ is the number of image dimensions, $R_{S S R, n}$ and $w_{n}$ are the scene reflectivity and weight of the nth scale (obtained by single-scale Retinex).

At the same time, in order to reduce the color shift generated by the MSR algorithm, an MSR color restoration (MSRCR) algorithm is proposed in [8]. Using the color recovery function to obtain the corresponding MSR reflectance and output reflectivity, the formula is:

$$
R_{\text {MSRCR }}(x, y)=H(x, y) \cdot R_{\text {MSR }}(x, y)
$$

Among them, $H(x, y)$ denotes the color recovery function.

\section{The Proposed Algorithm}

Retinex-based traditional image enhancement algorithms convert RGB colors to other spaces, such as HSV or YCbCr, and use Retinex to process the luminance channels. However, in the color spaces of $\mathrm{YCbCr}$ and $\mathrm{HSV}$, when the brightness changes, the associated chromaticity values also change accordingly. In addition, the number of color differences is not exactly the same as the number of differences perceived by the human visual system (HVS). In contrast, the lab color space alone changes the luminance and chrominance values to more faithfully reflect the human visual system. Therefore, we use the lab color space and only perform Retinex-based enhancements on the luma channel. In the chroma channel we still retain the original color information to avoid color shifts.

\subsection{Illuminance Estimation}

Based on the principle of the human visual system, we transform through the lab color space, and then according to the Retinex theory [1], we regard the observed intensity of the L-channel image as a combination of the relevant reflectivity and illuminance.

$$
I_{\text {lum }}(x, y)=R_{\text {lum }}(x, y) \cdot L_{\text {lum }}(x, y)
$$

Where, $R_{\text {lum }}(x, y), L_{\text {lum }}(x, y)$ and $I_{\text {lum }}(x, y)$ and the reflectance, illumination, and intensity of the pixel locations in the L-channel component, respectively. In addition, the reflectivity is normalized to $[0,1]$, so $I_{\text {lum }}(x, y) \leq L_{\text {lum }}(x, y)$.

Traditional algorithms based on Retinex estimate the illuminance by convolving the input image with a smoothing filter, such as Gaussian, bilateral filters, and pilot filters. The Gaussian filter 
calculates the average pixel value and therefore often produces halo artifacts. In contrast, bilateral filters can preserve edges in the input image and effectively reduce the generation of halo artifacts.

Note that traditional bilateral filtering assigns higher weights to neighboring pixels, which are geometrically closer and have more similar pixel values to a given target pixel. However, in some target pixels, due to the smoothness of the bilateral filter, the estimated illumination value is even smaller than the observed intensity value, resulting in an invalid reflectance value greater than one. In this case, it is additionally necessary to normalize or adjust the range of reflectance values, which may produce smooth pixel values in the enhanced image.

Then we use adaptive bilateral filtering to ensure the scope of reflection. Let $P(x, y)$ be a group of pixels that are adjacent to $(x, y)$ pixels. By selecting $P(x, y)$ pixels in the range, we get a set of $(u, v)$ pixels that have a similar color to the $(x, y)$ pixel and have a larger luminance value than the $(x, y)$ pixel. $S(x, y)$ expressed as:

$$
\begin{aligned}
S(x, y)=\{ & (u, v) \mid(u, v) \in P(x, y), \\
& \left.I_{\text {lum }}(u, v)>I_{\text {lum }}(x, y), d_{(x, y)}(u, v)<\mathrm{T}\right\}
\end{aligned}
$$

Among them, the expression $d_{(x, y)}(u, v)$ of the chromaticity distance between $(x, y)$ and $(u, v)$ is calculated by:

$$
\begin{aligned}
& d_{(x, y)}(u, v)= \\
& \sqrt{\left(I_{a}(x, y)-I_{a}(u, v)\right)^{2}+\left(I_{b}(x, y)-I_{b}(u, v)\right)^{2}}
\end{aligned}
$$

Where, $I_{a}$ and $I_{b}$ are the a and $\mathrm{b}$ channel components in the lab color space respectively, $\mathrm{T}$ indicating the set critical values, which we set $\mathrm{T}=10$ according to experience.

Then, the pixel's lighting estimate formula is:

$$
\begin{aligned}
& \hat{L}_{\text {lum }}(x, y)= \\
& \frac{\sum_{(u, v) \in S(x, y)} F_{\text {geo }}(u, v) \cdot F_{\text {int }}(u, v) \cdot I_{\text {lum }}(u, v)}{\sum_{(u, v) \in S(x, y)} F_{\text {geo }}(u, v) \cdot F_{\text {int }}(u, v)}
\end{aligned}
$$

Among them, $F_{\text {int }}(u, v)$ and $F_{g e o}(u, v)$ are respectively the intensity similarity and geometric similarity in bilateral filtering.

$$
\begin{gathered}
F_{\text {geo }}(u, v)=\frac{1}{2 \pi \sigma_{1}^{2}} \exp \left(-\frac{(x-u)^{2}+(y-v)^{2}}{2 \sigma_{1}^{2}}\right) \\
F_{\text {int }}(u, v)=\frac{1}{2 \pi \sigma_{2}^{2}} \exp \left(-\frac{\left(I_{\text {lum }}(x, y)-I_{\text {lum }}(u, v)\right)^{2}}{2 \sigma_{2}^{2}}\right)
\end{gathered}
$$

Among them, $\sigma_{1}=3$ and $\sigma_{2}=5$.

Finally, the formula of reflectivity can be obtained by formula:

$$
R_{\text {lum }}(x, y)=\frac{I_{\text {lum }}(x, y)}{\hat{L}_{\text {lum }}(x, y)}
$$

By increasing the histogram dynamic range of the estimated light image intensity $\hat{L}_{\text {lum }}$, we improved the contrast of the input image. This can be done using traditional histogram equalization or cumulative distribution functions (matching, however, these methods tend to produce unnatural images.

In this paper, we use a parabolic-based tone mapping curve. As shown in the transfer function region in Figure 1, we define a parabola curve on a straight line with a slope of $45^{\circ}$, and the apex of the parabola deviates from the blue line to reach $|\lambda|$. Therefore, the low intensity values of this parabola curve have fairly steep slopes, and the change in high intensity values is slow. $\lambda$ calculated by the following formula, we adaptively find a suitable parabolic curve for the input image. 


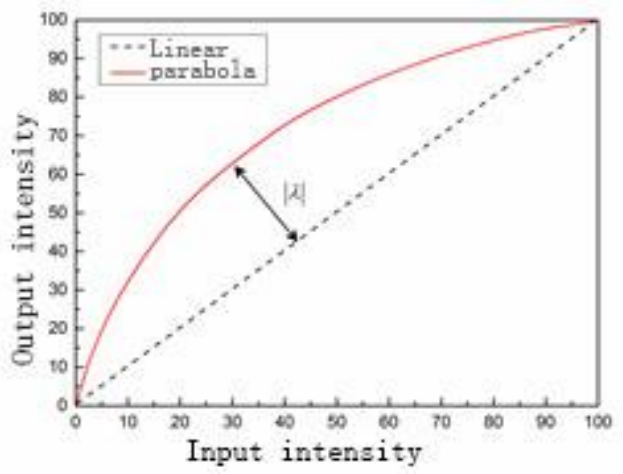

Figure 1. Intensity conversion function based on the parabola

\subsection{Gamma Function}

According to the perception principle of human vision, the image scene atmosphere has a greater influence on human vision. The algorithm in the previous section is only a global basis and does not apply to image details. This paper uses two-dimensional gamma function, namely spatial adaptive brightness transfer function. The main expression $\varphi$ of the image is expressed as follows:

$$
\varphi=e^{\frac{\sum_{x}^{m}=1 \sum_{y}^{n}=1 \rho(x, y)}{m \times n}}
$$

Among them, $\rho$ is the image represented by the logarithm and $m \times n$ represents the size of the image.

$$
\rho(x, y)=\log \{\varepsilon+\mathrm{B}(x, y)\}
$$

Among them, $L$ indicate the brightness of the image $\varepsilon$ is to avoid the appearance of black pixels.

The 2-dimensional gamma function formula is as follows:

$$
B_{m}(x, y)=B(x, y)^{\left(\frac{\Delta-\partial(x, y)}{\Delta}\right)^{\varphi}}
$$

Wherein, $B_{m}$ represents the base layer of the mapping.

The average brightness of the image $\Delta$ is expressed as follows:

$$
\Delta=\frac{1}{m \times n} \sum_{x}^{m}=1 \sum_{y}^{n}=1 B(x, y)
$$

In addition, the local area $\partial$ is expressed as follows:

$$
\partial(x, y)=\frac{1}{\Omega^{2}}\left[\sum_{q=y-\frac{\Omega}{2}}^{y+\frac{\Omega}{2}}\left(\sum_{p=x-\frac{\Omega}{2}}^{x+\frac{\Omega}{2}} B(p, q)\right)\right]
$$

Where, $\Omega$ indicates a local area, set to $25 \times 25$.

To avoid artifacts in the enhanced image, we will use the detail layer of the 2-dimensional gamma function mapping, combined with the global base layer generated in the previous section, expressed as follows:

$$
\begin{aligned}
& E_{R}(x, y)=B_{m}(x, y) \times D_{R}(x, y) \\
& E_{G}(x, y)=B_{m}(x, y) \times D_{G}(x, y) \\
& E_{B}(x, y)=B_{m}(x, y) \times D_{B}(x, y)
\end{aligned}
$$

Among them, $E(x, y)$ indicate the enhanced output image. The proposed algorithm flowchart is shown in Figure 2.

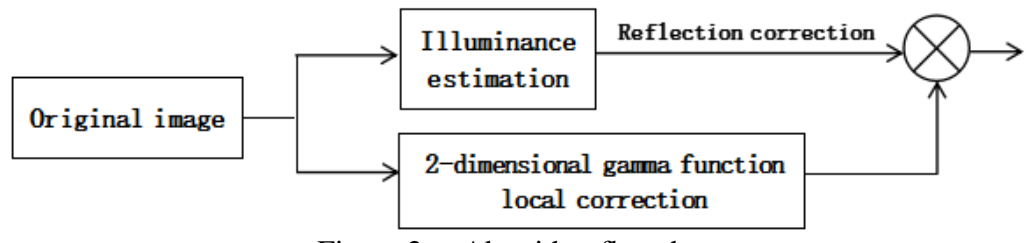

Figure 2. Algorithm flowchart 


\section{Experiment and Result Analysis}

In order to verify the performance of the proposed algorithm, the proposed algorithm is compared with other conventional enhancement algorithms $[9,10]$. The experimental platform is Windows 7 operating system, Intel(R) Core(TM) i7 CPU (3.00 GHz), 4GB memory and windows7 64-bit system, Matlab7.8 simulation environment. The experimental data set was 10 low-light images.

\subsection{Evaluation Index}

The evaluation index of the image enhancement algorithm is the brightness, standard deviation, and contrast increments, so as to make a concrete comparison of objective effects [10]. Brightness is the brightness of the image, calculated as follows:

$$
\bar{I}=\frac{1}{3 \times M \times N} \sum_{i=1}^{3} \sum_{m=1}^{M} \sum_{n=1}^{N} I(m, n, i)
$$

Where, $\mathrm{M}$ and $\mathrm{N}$ are the size of the image, $i$ is the number of channels.

The standard deviation is the degree of black-and-white contrast of the image. The larger the value, the better the observation by the human eye. The expression formula is as follows:

$$
\begin{aligned}
& s=\sqrt{\frac{1}{n} \sum_{i=1}^{n}\left(x_{i}-\bar{x}\right)^{2}}, \\
& \bar{x}=\frac{1}{n} \sum_{i=1}^{n} x_{i}
\end{aligned}
$$

The contrast increment is the ratio of the processed contrast to the original contrast, that is, the degree of contrast change. If the value is greater than 1, the contrast of the enhanced image is improved, and the expression formula is as follows:

$$
K=C_{\text {After processing }} / C_{\text {original }}
$$

Where, $C$ is the average of the local contrast within the window range is represented, and the sliding window size is $3 \times 3$.

The enhancement effect of the three algorithms is shown in Fig. 3. It can be seen that the algorithm of this paper retains the details of the image layout area higher and has better naturalness.

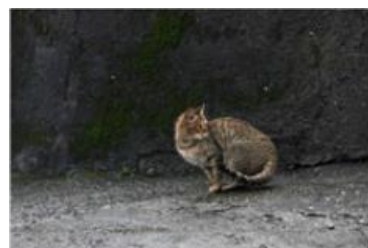

(a)

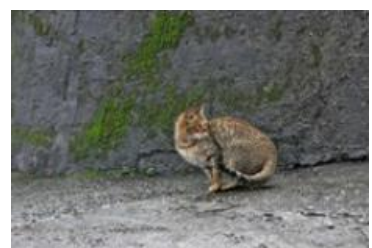

(c)

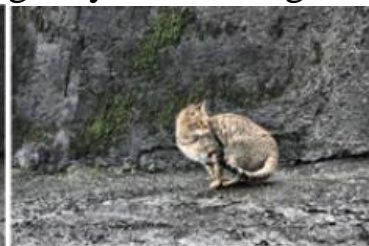

(b)

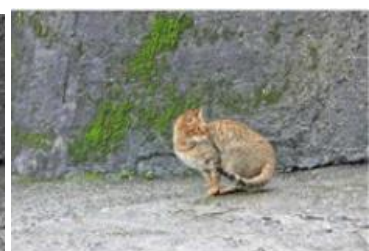

(d)

Figure 3. Image contrast enhancement effect: (a) low contrast input image, (b) enhanced image, (c) enhanced image, (d) proposed algorithm

\subsection{Objective Indicators for Enhancing Effectiveness}

Using 10 low-light images as experimental data sets, the algorithm proposed in this paper was enhanced experimentally. Through the three objective indicators of brightness, standard deviation, and contrast increment, the enhanced effect of the proposed algorithm was evaluated, as shown in Table 1. Show. 
Table 1. Average enhancement index

\begin{tabular}{|c|c|c|c|}
\hline Enhancement Method & Brightness & Standard deviation & Contrast increment \\
\hline literature[9] & 463.22 & 10.32 & 1.22 \\
\hline literature [10] & 472.54 & 10.52 & 1.25 \\
\hline This Algorithm & 493.13 & 10.45 & 1.29 \\
\hline
\end{tabular}

From Table 1, it can be seen that the contrast increments of the three methods are all greater than 1 , which means that the contrast of the image is effectively improved. Because this algorithm can adaptively apply bilateral filtering, and can choose the appropriate two-dimensional gamma function, the experimental results show that compared with the other two kinds of enhancement methods, the contrast increase effect of the proposed algorithm is obvious.

\section{Conclusions}

In this paper, a color Retinex image enhancement algorithm based on L-channel illuminance estimation and two-dimensional gamma function is proposed. The algorithm uses L-channel image intensity as the product of relative reflectivity and illuminance through Lab color space transformation. Bilateral filtering is used to estimate the global illumination intensity. Then, pixel mapping is performed using a two-dimensional gamma function to achieve adaptive adjustment of local details. Experimental results show that compared with other Retinex image enhancement algorithms, the proposed algorithm can maintain the local detail texture of the enhanced image better, with higher color constancy, higher contrast and no artifact.

\section{References}

[1] Shin K Y, Park Y H, Nguyen D T, et al. Finger-vein image enhancement using a fuzzy-based fusion method with Gabor and Retinex filtering. Sensors, 2014, 14(2):3095-129.

[2] Prabhu M, Rajarajan S, Karthikeyan M P. Implementing histogram equalization and retinex algorithms for image contrast enhancement. International Journal of Applied Engineering Research, 2014, 9(20):7311-7318.

[3] Jobson D J, Rahman Z, Woodell G A. Properties and performance of a center/surround retinex. IEEE Transactions on Image Processing A Publication of the IEEE Signal Processing Society, 1997, $6(3): 451-62$.

[4] Qin Xujia, Wang Huiling, DuYicheng,etl. Structured Light Image Enhancement Algorithm Based on Retinex in HSV Color Space. Journal of Computer-Aided Design \& Computer Graphics, 2013, 25(4):488-493.

[5] DU Ming, ZHAO Xiang-jun. Face Enhancement Algorithm with Variable Illumination Based on Improved Retinex. Computer Science, 2016, 43(2):105-108.

[6] Wang Y, Wang H, Yin C, et al. Biologically inspired image enhancement based on Retinex[J]. Neurocomputing, 2016, 177:373-384.

[7] Zhao Huaxia, Yu Jing, Xiao Chuangbai. Night Color Image Enhancement via Optinmization of Purpose and Improved Histogram Equalization, 2015, 52(6):1424-1430.

[8] Jiang B, Woodell G A, Jobson D J. Novel multi-scale retinex with color restoration on graphics processing unit. Journal of Real-Time Image Processing, 2015, 10(2):239-253.

[9] Tsai C M. Adaptive Local Power-Law Transformation for Color Image Enhancement. Applied Mathematics \& Information Sciences, 2013, 7(5):2019-2026.

[10] Nnolim U A. Log-hybrid architecture for tonal correction combined with modified un-sharp masking filter algorithm for colour image enhancement. Integration the Vlsi Journal, 2014, 48(1):221-229. 